\title{
One Method for Low Elevation Estimation Based on Unitary transformation in MIMO Radar
}

\author{
De-wang Wang ${ }^{1,2}$, Jin-liang Guo $^{2}$, Ze-long Li $i^{2}$,Jian-hong Yang ${ }^{1^{*}}$ \\ ${ }^{1}$ School of Physical Science and Technology, Lanzhou University, Lanzhou 730000, China \\ ${ }^{2}$ Luoyang Electronic Equipment Test Center, Luoyang 471003, China
}

Keywords: MIMO radar; Multipath; DOA; Fading coefficient; Unitary transformation

\begin{abstract}
Multipath effection seriously affect the low elevation Angle estimation in MIMO radar,This paper Establish the multipath signal model , and put forward a kind of secondary general MUSIC algorithm based on unitary transformation (DU-G_MUSIC). First Based on general MUSIC algorithm, a joint estimation algorithm of elevation angle and multipath fading coefficient about target is proposed, In order to reduce the computational complexity, Avoiding search a wide range of angles, Then use the characteristics of the unitary transformation matrix, structure of real number domain data, get the initial estimation of low elevation Angle, And on the basis of initial estimates to obtain the real value of multipath fading coefficient, finally the DU-G_MUSIC algorithm on the real number field is used to estimate the optimized low elevation values. Due to multipath fading coefficient combined with Angle estimation, greatly improve the efficiency of the operation, to avoid the tedious decorrelation smoothing procedure, get high Angle resolution, on the premise of no loss of array aperture, reduces to the special requirements of array structure, has high engineering value.
\end{abstract}

\section{Introduction}

New system of multiple input multiple output (MIMO) radar, is a hotspot in research of radar at home and abroad, In recent years, many scholars study the characteristics of MIMO radar from different angles ${ }^{[1]-[3][9][10]}$. because of the wide beam, makes the multipath signal produced by intensifying, On the other hand make use of orthogonal waveform, At the same time bring frequency diversity and space diversity, Can reduce the probability of multipath signal cancellation, effectively suppress multipath clutter interference. The transmission and reception multipath MIMO radar needs to consider at the same time, after dealing with the receiver matched filter effective channel number is $\mathrm{M}$ times that of the conventional radar, dealing with computational complexity. Therefore, based on the decorrelation processing algorithms cannot be directly applied to the multipath channel model of MIMO radar Angle estimation. By improving the super resolution algorithm can effectively solve the problem of covariance matrix rank loss, but will reduce the effective aperture antenna, reduce angular resolution. To do this, such as generalized MUSIC algorithm is presented to directly solve multipath, coherent Angle estimation problem by Zoltowski Et al ${ }^{[4]}$

For the multipath MIMO radar low elevation Angle estimation under the background of the research is relatively small, Literature ${ }^{[5]}$ proposed one method based on maximum likelihood estimation, need spectrum peak search, computational complexity. By means of matrix of beam characteristics, effectively overcome the multipath effect, achieve low elevation Angle estimation ${ }^{[6]}$. Low elevation algorithm was proposed based on the beam space, greatly simplify the calculation, make full use of the MIMO radar beam characteristics ${ }^{[7]}$. One algorithm is presented based on the quadratic differential pretreatment, this algorithm has nothing to do with the signal environment, has a good robustness ${ }^{[8]}$. Above the low elevation Angle estimation algorithm for MIMO radar are conducted on complex domain, Angle estimation computation is lager.

This paper in the complex domain complete the joint estimation of incident Angle and the multipath attenuation coefficient with generalized MUSIC algorithm, the Angle measuring precision is improved greatly. In order to further reduce the computational complexity, avoid search a wide range of perspectives, Then based on the unitary transformation matrix characteristics, structure the 
data matrix for real number domain, get the multipath Angle initial estimation. And on the basis of initial estimates to get the multipath attenuation coefficient for real number domain, put forward a kind of secondary general MUSIC algorithm based on unitary transformation (DU-G_MUSIC). Due to multipath fading coefficient combined with Angle estimation, greatly improve the efficiency of the operation, to avoid the tedious decorrelation smoothing procedure, get high Angle resolution, on the premise of no loss of array aperture, reduces to the special requirements of array structure, has high engineering value.

\section{The signal model}

MIMO radar is a new system radar, launch multiple orthogonal signal, have multiple channels. So not only need to consider accepting multipath, but to consider multipath launch, As shown in figure 1.

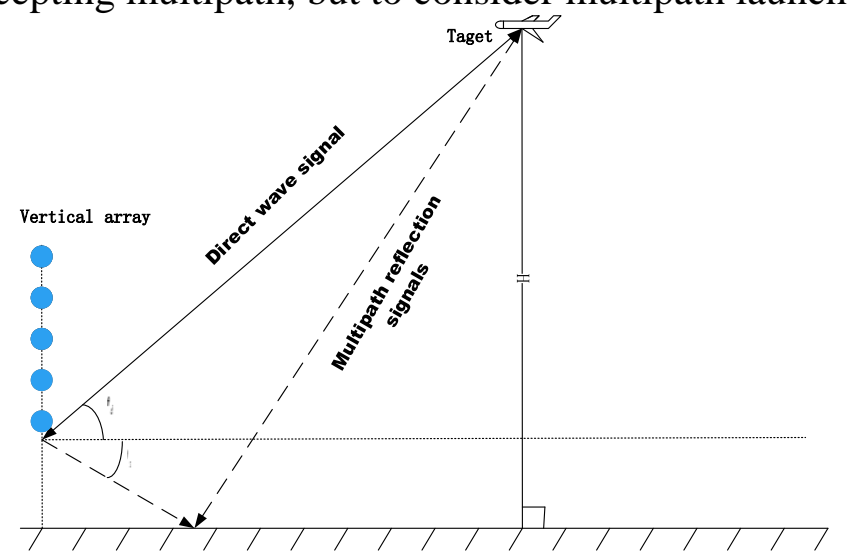

Figure 1 the model for the MIMO radar multipath specular reflection

Assume that each channel to launch $\mathrm{M}$ orthogonalsignal: $S_{1}(\mathrm{t}), \ldots S_{m}(\mathrm{t}), \ldots S_{M}(\mathrm{t})$, and meet:

$$
\int_{t_{i}}^{t_{i}+\tau_{p}} S_{i}(\mathrm{t}) S_{j}^{*}(\mathrm{t}) \mathrm{dt}= \begin{cases}c & i \neq j \\ 0 & i=j\end{cases}
$$

Among them, i $\mathrm{j}$ is a number of different transmission channel signal. Assume that reflection coefficient is changeless, The nTH receiver array receives the baseband signal for:

$$
\begin{aligned}
& x_{n}(\mathrm{t})=K\left[a_{n}\left(\theta_{d}\right)+\xi^{r} a_{n}\left(\theta_{s}\right)\right] \\
& {\left[a_{t}^{T}\left(\theta_{d}\right)+\xi a_{t}^{T}\left(\theta_{s}\right)\right] \cdot \mathrm{G}+n_{n}(\mathrm{t})}
\end{aligned}
$$

Among them, $\mathrm{K}$ is a composite coefficient of target scattering, time delay, doppler related, Multipathattenuation coefficient $\xi=\rho e^{-j 2 \pi f_{0} \Delta \tau}$, The ground reflection coefficient $\rho$, Launch direct wave Steering vector $a_{t}\left(\theta_{d}\right)$, The reflected wave Steering vector $a_{t}\left(\theta_{s}\right)$.

After Matched filtering, The array signal as follows:

$$
\begin{aligned}
Z & =\left[\mathrm{z}_{1}(\mathrm{t}), \mathrm{z}_{2}(\mathrm{t}), \ldots \mathrm{z}_{n}(\mathrm{t}) \ldots \mathrm{z}_{N}(\mathrm{t})\right]^{T} \\
& =A \cdot S+N(\mathrm{t})
\end{aligned}
$$

$A=\left[a_{r}\left(\theta_{d}\right) \quad a_{r}\left(\theta_{s}\right)\right] \otimes\left[a_{t}\left(\theta_{d}\right) a_{t}\left(\theta_{s}\right)\right], S=\left[1, \xi, \xi, \xi^{2}\right]^{T}$, Noise vector is zero gaussian distribution.

\section{Theoretical analysis and algorithm implementation}

The covariance matrix eigenvalue decomposition is:

$$
\mathbf{R}_{Z}=\mathbf{U}_{Z s} \boldsymbol{\Sigma}_{Z s} \mathbf{U}_{Z s}^{H}+\mathbf{U}_{Z N} \boldsymbol{\Sigma}_{Z N} \mathbf{U}_{Z N}^{H}
$$

Due to the coherence of multipath reflection signals and target directly reflect the direct wave signal, There is only a larger eigenvalues.

According to the generalized MUSIC thoughts, Construct the spatial spectrum function is: 


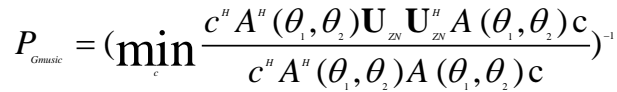

$$
\begin{aligned}
& A\left(\theta_{1}, \theta_{2}\right)=\left[a_{r}\left(\theta_{1}\right) \quad a_{r}\left(\theta_{2}\right)\right] \otimes\left[a_{t}\left(\theta_{1}\right)\right.
\end{aligned}
$$

$\left.a_{t}\left(\theta_{2}\right)\right], c=\left[\begin{array}{llll}1 & \Gamma & \Gamma & \Gamma^{2}\end{array}\right]^{T}, \Gamma$ is an unknown parameters, Equivalent to the following equations:

$$
\operatorname{det}\left\{\begin{array}{l}
A^{H}\left(\theta_{1}, \theta_{2}\right) \mathbf{U}_{Z N} \mathbf{U}_{Z N}^{H} A\left(\theta_{1}, \theta_{2}\right) \\
-\gamma A^{H}\left(\theta_{1}, \theta_{2}\right) A\left(\theta_{1}, \theta_{2}\right)
\end{array}\right\}=0
$$

make $\square=A^{H}\left(\theta_{1}, \theta_{2}\right) \mathbf{U}_{Z N} \mathbf{U}_{Z N}^{H} A\left(\theta_{1}, \theta_{2}\right)$, According to the nature of the determinant : If and only if $\square$ is Singular, $\gamma=0$ is solution of the equations.

Therefore, to construct revised spatial spectral function :

$$
P_{\text {Gmusic }}=\frac{\operatorname{det}\left\{A^{H}\left(\theta_{1}, \theta_{2}\right) A\left(\theta_{1}, \theta_{2}\right)\right\}}{\operatorname{det}\left\{A^{H}\left(\theta_{1}, \theta_{2}\right) \mathbf{U}_{z N} \mathbf{U}_{z N}^{H} A\left(\theta_{1}, \theta_{2}\right)\right\}}
$$

Define the noise subspace projection matrix $P_{Z N}=\mathbf{U}_{Z N} \mathbf{U}_{Z N}^{H}$, Based on the subspace orthogonality principle are:

$$
P_{Z N}(\mathrm{AS})=0
$$

Estimates of the multipath attenuation coefficient is:

$$
\hat{\xi}=\arg \min _{\xi}\left\|\mathbf{U}_{Z N}^{H} A q\right\|
$$

$q=\left[\begin{array}{llll}1 & \xi & \xi & \xi^{2}\end{array}\right]^{T}, \quad \mathfrak{I}=[1,0,0,0]^{T}$, and satisfy the relationship:

$$
q^{H} \mathfrak{I}=1
$$

Based on constrained least squares method can find out:

$$
q=\frac{\left\{A^{H} \mathbf{U}_{Z N} \mathbf{U}_{Z N}^{H} A\right\}^{-1} \mathfrak{I}}{\mathfrak{I}^{H}\left\{A^{H} \mathbf{U}_{Z N} \mathbf{U}_{Z N}^{H} A\right\}^{-1} \mathfrak{I}}
$$

If known direct Angle and multipath reflection Angle, according to Equation.11 multipath attenuation coefficient can be concluded, In the actual we can't get exactly multipath attenuation coefficient, So by the following spectral function to estimate multipath attenuation coefficient value:

$$
Q=\frac{\left\{A^{H}\left(\theta_{1}, \theta_{2}\right) P_{Z N} A\left(\theta_{1}, \theta_{2}\right)\right\}^{-1} \mathfrak{I}}{\mathfrak{I}^{H}\left\{A^{H}\left(\theta_{1}, \theta_{2}\right) P_{Z N} A\left(\theta_{1}, \theta_{2}\right)\right\}^{-1} \mathfrak{I}}
$$

At the same time structure joint spectrum function:

$$
P_{\text {GAmusic }}=\frac{Q^{H} A^{H}\left(\theta_{1}, \theta_{2}\right) A\left(\theta_{1}, \theta_{2}\right) Q}{Q^{H} A^{H}\left(\theta_{1}, \theta_{2}\right) P_{\text {ZN }} A\left(\theta_{1}, \theta_{2}\right) Q}
$$

When $\theta_{1}=\theta_{d}, \theta_{2}=\theta_{s}$, The value of $\mathrm{Q}$ is the estimates of multipath attenuation coefficient, and the spatial spectrum function is peak.

\section{The improvement of algorithm}

The above based on the generalized MUSIC spatial spectrum estimation algorithm of low elevation are in complex domain, computational cost is larger, it is not conducive to the engineering application. By using the properties of unitary transformation matrix, Any Centro-Hermitian matrix after unitary transformation to be a real matrix. Can consider to expand the ideas of the generalized MUSIC to the real number field. $\mathbf{R}_{Z}$ is Hermitian matrix,but is not Centro-Hermitian matrix, So will transform it to the real number field. accept data use two-way smoothing, namely:

$$
R_{Z b}=\frac{1}{2}\left(R_{Z}+J_{N M} R_{Z}^{*} J_{N M}\right)
$$


$J_{N M}$ is one $N M \times N M$ displacement matrix.

Define the unitary transformation matrix:

$$
\begin{aligned}
& \widetilde{\sigma}_{2 l}=\frac{1}{\sqrt{2}}\left[\begin{array}{ll}
I_{l} & j I_{l} \\
J_{l} & -j J_{l}
\end{array}\right] \\
& \mho_{2 l+1}=\frac{1}{\sqrt{2}}\left[\begin{array}{lll}
I_{l} & 0 & j I_{l} \\
0 & \sqrt{2} & 0 \\
J_{l} & 0 & -j J_{l}
\end{array}\right]
\end{aligned}
$$

The smoothing covariance matrix after unitary transformation to obtain:

$$
\begin{aligned}
R_{\mho} & =\mho^{H} R_{Z j} \circlearrowright \\
& =\frac{1}{2} \mho^{H}\left(R_{Z}+J_{N M} R_{Z}{ }^{*} J_{N M}\right) \mho \\
& =\operatorname{Re}\left(\mho^{H} R_{Z} \circlearrowright\right)
\end{aligned}
$$

Make $A_{v}\left(\theta_{1}, \theta_{2}\right)=\mho^{H} A\left(\theta_{1}, \theta_{2}\right)$, noise subspace projection matrix of Real number domain is $P_{\mathrm{ZNU}}=\mathbf{U}_{\mathrm{ZNU}} \mathbf{U}_{\mathrm{ZNU}}^{H}$, Get a real number domain search space spectrum :

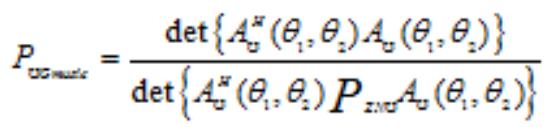

Initial estimates remember to $\left(\theta_{d 1}, \theta_{s 1}\right)$, In order to further improve Angle measuring accuracy, According to Equation.12:

$$
\begin{aligned}
& Q_{r}=\frac{\left\{A_{v}^{H}\left(\theta_{d 1}, \theta_{t 1}\right) P_{z v U} A_{v}\left(\theta_{d 1}, \theta_{t 1}\right)\right\}^{-1} \mathfrak{I}}{\mathfrak{S}^{H}\left\{A_{U}^{H}\left(\theta_{\Delta 1}, \theta_{i 1}\right) P_{z N v} A_{U}\left(\theta_{A 1}, \theta_{t 1}\right)\right\}^{-1} \mathfrak{I}} \\
& Q_{r}=\left[\begin{array}{llllll}
1 & \xi_{r} & \hat{\xi}_{r} & \hat{\xi}_{r}^{2}
\end{array}\right]^{T}, \hat{\xi}_{r} \text { is estimation of Real multipath attenuation coefficient, make constraint to }
\end{aligned}
$$
range of search Angle by $\left(\theta_{d 1}, \theta_{s 1}\right)$, Might as well take the search range for:

$$
\begin{aligned}
& \theta_{1} \in\left[\theta_{d 1}-5 \operatorname{std}\left(\theta_{d 1}\right), \theta_{d 1}+5 s t d\left(\theta_{d 1}\right)\right] \\
& \theta_{2} \in\left[\theta_{s 1}-5 \operatorname{std}\left(\theta_{s 1}\right), \theta_{s 1}+5 \operatorname{std}\left(\theta_{s 1}\right)\right]
\end{aligned}
$$

The second Angle estimation is:

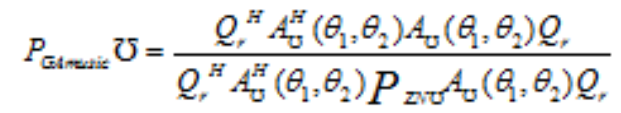

The resulting multipath incidence estimate to remember $\left(\theta_{d 2}, \theta_{s 2}\right)$, it is based on the unitary transformation of the secondary generalized MUSIC algorithm,call it DU-G_MUSIC.

\section{The simulation}

The simulation environment:MIMO radar, Sending and receiving arrays are 8 arrays vertical line array and interval is half wavelength , Height of antenna erection is $16 \mathrm{~m}$, Aim at $1000 \mathrm{~m}$, Multipath reflection coefficient is $\rho=0.9 e^{j \pi}$, direct Angle is $4.57^{\circ}$,multipath reflection Angle is $-4.607^{\circ}$, Radar transmitting orthogonal coded signal, Carrier frequency is 3GHZ, Pulse repetition interval is $1 \mathrm{~ms}$, Signal duty ratio is $80 \%$, Transmitted pulse count is 256, SNR $15 \mathrm{~dB}$. The simulation results are as follows:

Figure 2 is a curve of one dimensional Angle spectrum searching, Existed not only direct wave incident Angle, also reflected wave incident Angle, the equivalent of two highly coherent goal, so you can see two peaks. Under the condition of the same parameters, Compared to phased array radar (PA) the MIMO radar has better Angle measurement properties, the Angle estimation precision is 
high. Thanks to the orthogonal signal frequency diversity and space diversity, Can improve the probability of multipath signal cancellation, Effectively suppress multipath clutter interference.

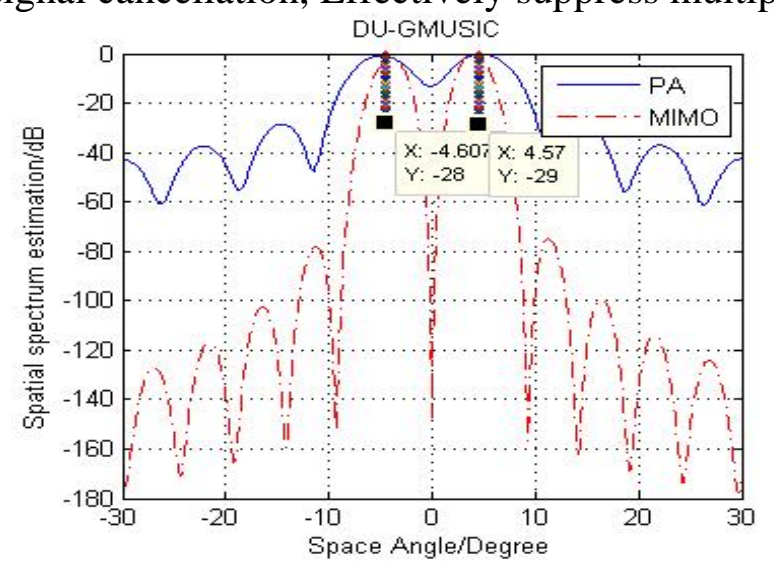

Figure 2 One dimensional search spectrum curve

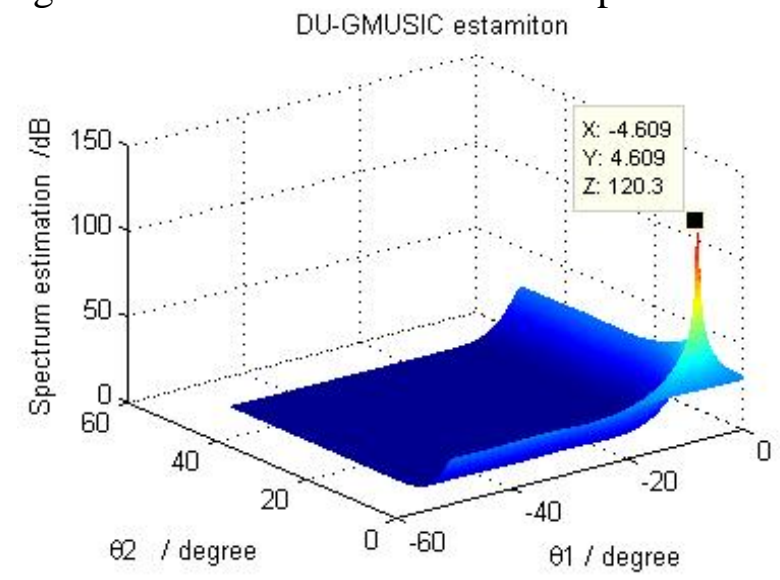

Figure 3 Two dimensional search space spectrum

Figure 3 is the corresponding two-dimensional search space spectrum, clearly see the use of the improved DU-GMUSIC algorithm has the very good resolution multipath Angle, Can correctly estimate low elevation, The simulation results and the value is very close, the rationality of the theory is verified further.

In order to better estimate the validity of the measured Angle, Define root mean square error of a single target launch Angle and acceptance Angle joint estimation is:

$$
\operatorname{RMSE}\left(\theta_{d}, \theta_{s}\right)=\sqrt{\frac{1}{\Pi} \sum_{i=1}^{\Pi}\left(\theta_{d}-\hat{\theta}_{d i}\right)^{2}+\left(\theta_{s}-\hat{\theta}_{s i}\right)^{2}}
$$

$\Pi$ is Monte-Carlo simulation times.

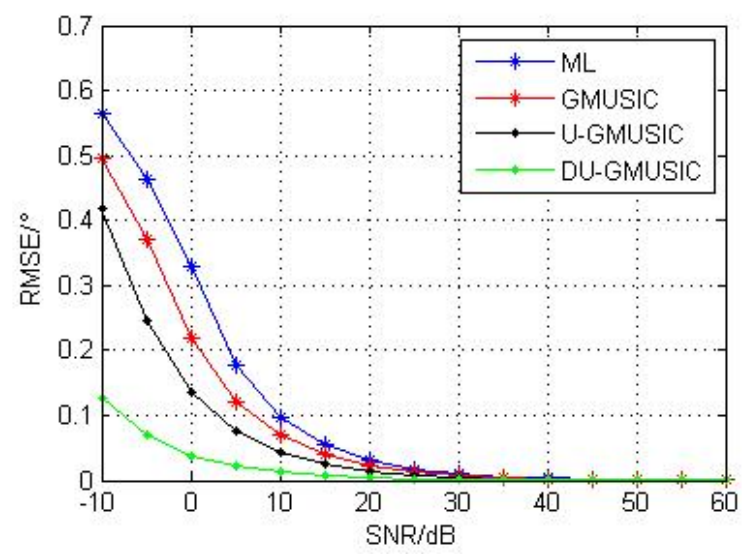

Figure 4 Comparison for the root mean square error of the Angle estimation with different algorithms Figure 4 is the simulation results with 300 Monte-Carlo simulation times, compares the change of the relationship of Root mean square error with the signal-to-noise ratio by different low elevation 
Angle estimation algorithm. Under the condition of the same parameter Settings, In low signal-to-noise ratio, Three kinds of algorithm based on generalized MUSIC thought its Angle estimation precision is higher than the method based on maximum likelihood theory, And root mean square error is minimum of secondary generalized MUSIC algorithm based on the unitary transformation, Make full use of the real number domain joint estimation of multipath attenuation coefficient and Angle reduces the search range of angles, and reduces the computational complexity. With the enhancing SNR, Several algorithms of estimation accuracy is almost the same, Thanks to the high signal-to-noise ratio of array signal processing efficiency, The difference decrease between different algorithms.

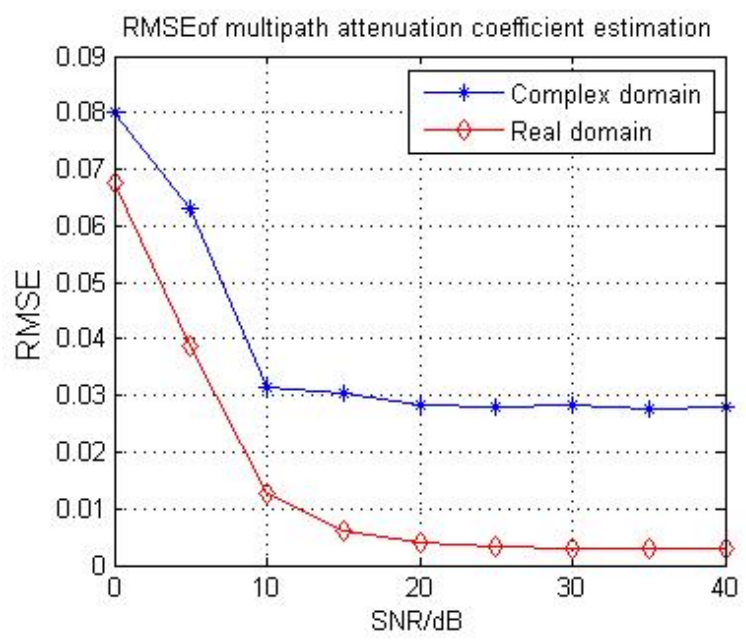

Figure 5 Root mean square error of multipath attenuation coefficient estimates

In Figure 5 ,It can be seen that the root mean square error of real number field is lower than the complex domain through joint space spectrum function of multipath attenuation coefficient estimates, Mainly because of real number domain space narrow spectral function estimation, thus improve the estimation precision.

\section{Summary}

DU-G_MUSIC algorithm,combine multipath attenuation coefficient with angle estimation in the real domain,Greatly improve the efficiency of the operation, Avoiding the tedious decorrelation smooth processing procedures, getting a higher low elevation resolution, On the premise of no loss of array aperture, Reduce the special requirements of array structure, has high engineering value.

\section{References}

[1] T Nizar, M K Hyuck.2004. Conjugate ESPRIT (C-SPRIT)[J]. IEEE Transactions on Antennas and Propagation. 52(10):2618-2624.

[2] Juan Zhang, Linrang Zhang, Nan Liu. 2009.An Efficient DOA Estimation Algorithm of Coherent Sources for MIMO Radar[C]. Processing of the IET International Radar Conference.

[3]T J Shan, Han M Wax, T Kailath. 1985.On spatial Smoothing for Direction of Arrival Estimation of Coherent Signals[J]. IEEE Transactions on Acoustic, Speech and Signal Processing. 34(4):806-811.

[4] Zoltowski M, Haber F.1986. A vector space approach to direction finding in a coherent multipath environment[J].IEEE Transaction on Antennas and Propagati.34(9):1069-1079.

[5]Xiang dong Wu,Yong bo Zhao,Shou hong Zhang.2008.a new method of doa estimation in MIMO radar low-angle tracking environment[J].Journal of xi 'an university of electronic science and technology.35(5):793-798. 
[6]Rong Xie,Zheng Liu,Jun Liu.2011.One fast estimation method of low elevation Based on the matrix of the MIMO radar beam[J].Journal of electronics and information.33(8):1833-1838.

[7]Jun Liu,Zheng Liu,Rong Xie.2011.Beam space Domain Angle estimation algorithm in VHF MIMO radar[J].Electronicjournals.39(9):1962-1966.

[8]Wei Bo Fu,Tao Su,Yong Bo Zhao.2013.A method for low elevation Angle estimation in MIMO radar[J]. Systems engineering and electronics.39(9):1962-1966.

[9] Wei Zhu and Bai-xiao Chen..2013.Altitude measurement based onterrain matching in VHF array radar[J].Circuits,Systems,and Signal Processing.32(2): 647-662.

[10] Wei Wang, Xian-peng Wang, Xin Li.2013.DOA estimation for monostatic MIMO radar based on unitary root-MUSIC[J].International Journal of Electronics.100(11):1499-1509.

\section{Acknowledgments}

The email of First author:

wangdew006@126.com

The email of Second author:

147688033@qq.com

The email of Communication author:

yangjh@lzu.edu.cn 